ORIGINAL ARTICLE

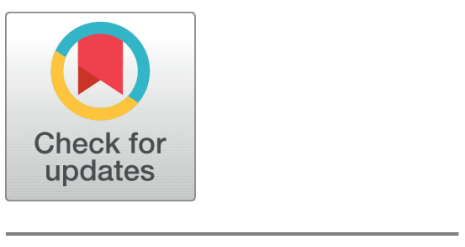

OPEN ACCESS

Received: 03-04-2020

Accepted: 23-04-2020

Published: 22-05-2020

Editor: Dr. Natarajan Gajendran

Citation: Kumar K, Wagan AA, Khuhro MA, Umrani A, Chhajro A, Hafeez A, Laghari AA (2020) Texture based FACE recognition using GLCM and LBP schemes. Indian Journal of Science and Technology 13(13): 1401-1411. https://doi.org/ 10.17485/IJST/v13i13.118

* Corresponding author. Asif Ali Wagan

Department of Computer Science, Sindh Madressatul Islam University, Karachi

asif.wagan@smiu.edu.pk

Funding: None

Competing Interests: None

Copyright: (c 2020 Kumar, Wagan, Khuhro, Umrani, Chhajro, Hafeez, Laghari. This is an open access article distributed under the terms of the Creative Commons Attribution License, which permits unrestricted use, distribution, and reproduction in any medium, provided the original author and source are credited.

Published By Indian Society for Education and Environment (iSee)

\section{Texture based FACE recognition using GLCM and LBP schemes}

\author{
Kamlesh Kumar ${ }^{1}$, Asif Ali Wagan ${ }^{1 *}$, Mansoor Ahmed Khuhro ${ }^{1}$, Aamir \\ Umrani ${ }^{1}$, Ameen Chhajro ${ }^{1}$, Abdul Hafeez ${ }^{1}$, Asif Ali Laghari ${ }^{1}$ \\ 1 Department of Computer Science, Sindh Madressatul Islam University, Karachi
}

\section{Abstract}

Objectives: Automatic face recognition has been an important area of biometric authentication and verification system in various applications including crime detection, access control, video surveillance, tracking service and other related area. Methods/Statistical analysis: In this study, we present Grey Level Co-occurrence Matrix (GLCM) over Local Binary Patterns (LBP) named as GOL texture feature technique for face classification. The experiments have been conducted on AT \& T Cambridge Laboratory face images also called (ORL-faces) and Georgia Tech (GT-faces) databases respectively. Findings: We performed comparative analysis of GLCM and LBP method separately and results showed that proposed GOL method outperformed in terms of average sensitivity, average specificity, and retrieval time. These findings show efficacy of our proposed system.

Keywords: GLCM; LBP; Face recognition; Feature extraction

\section{Introduction}

Since last two decades, automated face recognition remains a most challenging research area in the field of computer vision, human computer interaction and pattern recognition. Human face provides a unique information, which is required in various tasks, such as human crime detection, personal verification for access control, video surveillance for individual person identification, tracking service for monitoring employee's hours and besides other related scenarios ${ }^{(1-4)}$. Until now various face recognition systems have been suggested by different researchers. However, these systems fail to recognize human face in uncontrolled environment, such as small changes due to illumination, pose variation, facial expression and view point ${ }^{(5)}$. Therefore, it is difficult to develop a face recognition system which could provide high accuracy with a good retrieval of time. Typically, a face recognition system is divided into three modules: 1) image capture, 2) feature extraction and 3) classification. Figure 1 depicts the algorithm work flow. 


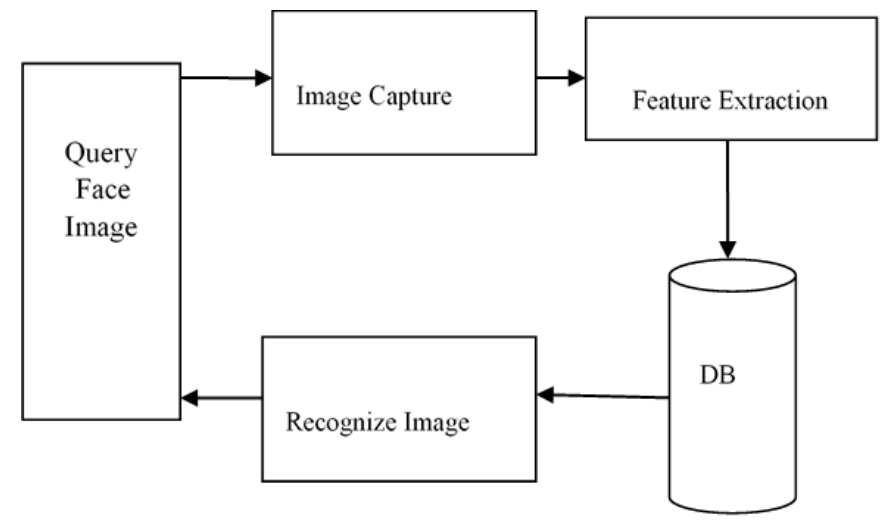

Fig 1. Face recognition system

As shown above, initially face image is given as input to recognition system and then image is captured by the biometric sensor module. After that feature extraction is carried out for the face image. Finally, feature matching is achieved from database images to recognize the face image.

The aim of this research work is to classify face images using texture features. For this purpose, GLCM and LBP texture methods have been used to extract statistical and structural features. Also, they are combined together to form a new method named as GOL. Similarly, they are tested for face images databases separately. The rest of this paper is arranged as follows. Section 2 presents the literature review related to face recognition system. Section 3 introduces the methodology of the proposed method. Section 4 illustrates the experimental results and discussion. Section 5 evaluates the performance of face recognition method on the databases. Section 6 provides the conclusion and future work.

\section{Literature Review}

The past face recognition techniques have been proposed based on color and texture features. Madan et al. ${ }^{(6)}$ provided a comprehensive overview on face recognition technique and also suggested future directions to work in this area. Face recognition system using texture features was proposed by Ahonen et al. ${ }^{(7)}$, in which a face image is divided into blocks and from each block LBP texture features are extracted. Then these features are combined together to represent global image descriptors. Similarly, face recognition using invariant features was suggested by Li et al. ${ }^{(8)}$ in which near infrared image device was implemented to minimize illumination effect on face images. For texture features, LBP method was used with Adaboost learning. Moreover, Hadid and Pietikäinen ${ }^{(9)}$ implemented spatiotemporal LBP for dynamic texture analysis for face gender classification from video sequences. Face detection from color images was suggested by Phuong-Trinh et al. ${ }^{(10)}$, where LBP was used for texture features for local and global shape analysis and also spatial descriptors from face images.

GLCM for face recognition was presented by Eleyan and Demirel ${ }^{(11)}$, in which fourteen Haralick were employed on face images that describe the pixel intensities as well as relative position of neighboring pixels for classification. Furthermore, extraction of color and texture for face images was investigated by Baek et al. ${ }^{(12)}$, authors suggested the part based head and shoulder detection which was obtained by skin color and LBP texture features. Moreover, Liao et al. ${ }^{(13)}$ proposed face recognition using multi scale binary pattern, this involves dividing an image into multi blocks and then location information related to each block for obtaining pixels intensities was achieved. In ${ }^{(14)}$ illustrated graph based approach for face recognition where a reference face graph (RFG) technique was used to recognize unknown face from the database. Whereas some researchers have suggested deep learning and neural network approaches for face recognition ${ }^{(15-19)}$

\section{Proposed scheme}

In this paper, two algorithms namely: GLCM and LBP have been implemented separately for face recognition. And they are combined together to obtain a fused GOL method as shown in Figure 2. The main algorithm steps of face recognition are:

Step 1: Firstly, a query face image is inputted to the system.

Step 2: Then pre-processing techniques are applied including image normalization and color space conversion.

Step 3: Face image texture features are extracted using LBP and GLCM method and also with combined GOL method.

Step 4: Finally, obtained results from each methods are compared with each other. 


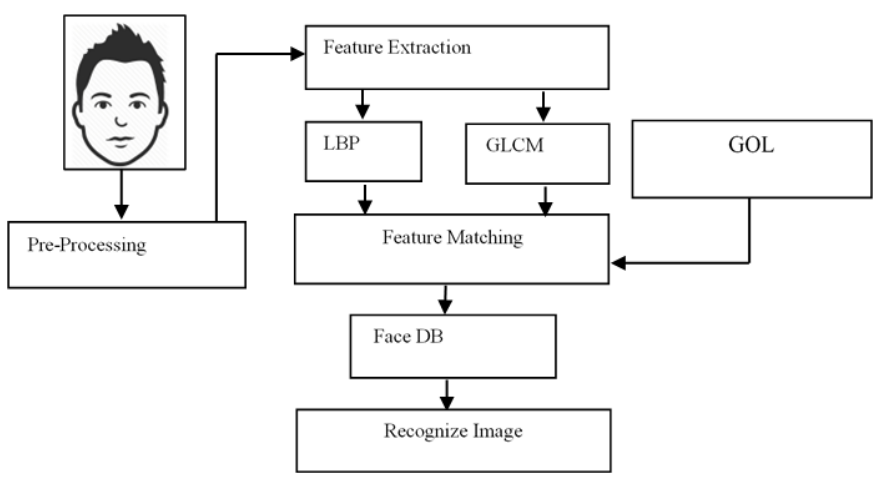

Fig 2. Algorithm for face recognition

\subsection{Face feature extraction}

Automatic face recognition employs Digital Image Processing and machine learning techniques. And they are classified into four types: Holistic or Appearance based, Template based, Part based, and Feature based ${ }^{(20)}$. This paper presents feature based approach for face recognition using GLCM and LBP texture methods.

\subsubsection{Local Binary Patterns (LBP) $m$ ethod}

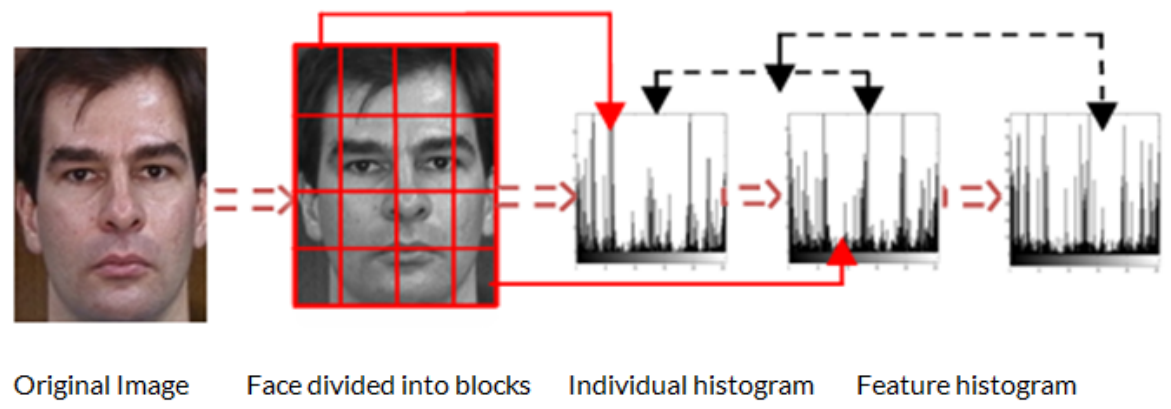

Fig 3. BP for Face Description

LBP is a technique for texture classification, which was first proposed by Ojala et al. ${ }^{(21)}$, and successfully used in real time face recognition. Also, this algorithm is computationally inexpensive because it describes the gray scale contrast and provides local spatial pattern for two- dimensional as well for image texture analysis this has been shown in Figure 3.

Initially face image is converted into gray scale and divided into 16 non-overlapping equal blocks. A $3 \times 3$ matrix is applied over each block. Then LBP histogram is generated from each block separately to describe the local texture features. Finally, features are combined together global feature histogram of face image.

\subsection{Gray Level Co-Occurrence Matrix (GLCM) method}

GLCM is a statistical method for texture analysis, which was first proposed by Haralick et al. ${ }^{(22)}$. It highlights the image texture by the computation of co-occurrence pairs of pixels those having unique value with respect to specified spatial relationship. And this relation is obtained on different offsets and angles. In Figure 4, color image is converted into gray scale and divided into 16 equal overlapped blocks. The statistical moments such as entropy, energy, contrast and homogeneity are drawn from each block, which eventually gives the local view of texture features. Then each feature is concatenated together as to provide the feature vector of GLCM image. 


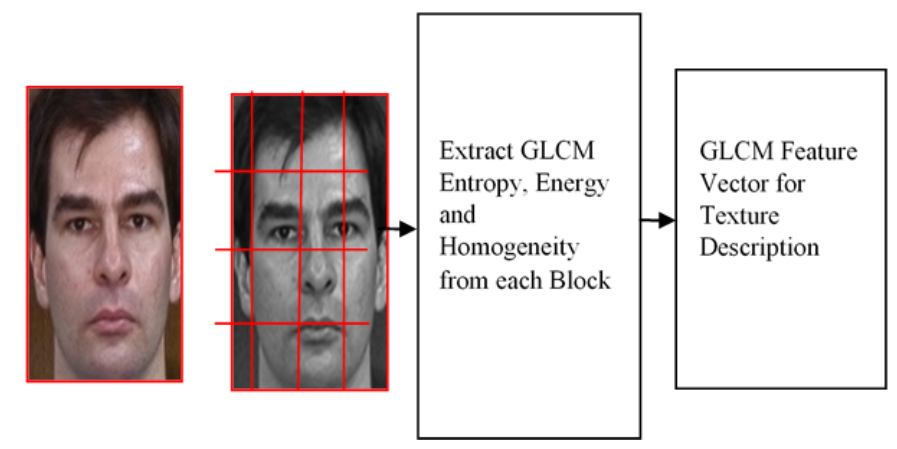

Fig 4. GLCM for face description

\subsubsection{Proposed Method}

In this section, proposed method is explained with implementation of GLCM over LBP. As shown in Figure 3 extracted LBP feature face image is further treated with GLCM. As a result they represent structural and statistical feature for face texture classification. Similarly, in Figure 5 obtained LBP image is further divided into 16 equal sized overlapping blocks. And then GLCM statistical moments are drawn from each block. These are entropy, energy, contrast, and homogeneity. Finally, obtained features from each block are fused together, resulting in global texture image feature.

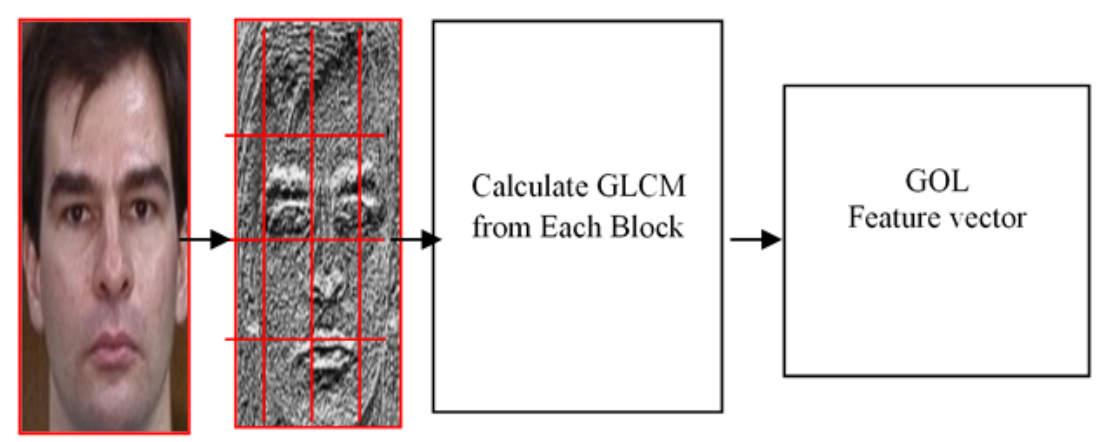

Fig 5. GOL feature image descriptor

\subsection{Evaluation metrics for face recognition}

Accuracy of developed face recognition system has been evaluated through statistical measures, these are sensitivity and specificity as shown in Equation (1) and (2). Efficiency of the system is also checked with retrieval time. However, similarity between query face image and face images in the database have been compared with Euclidean distance.
Sensitivity $=\mathrm{TP} /(\mathrm{TP}+\mathrm{FN})$
Specificity $=\mathrm{TN} /(\mathrm{TN}+\mathrm{FP})$

In Equation $(1 \& 2)$ represents, $\mathrm{TP}=$ true positive, $\mathrm{FN}=$ false negative, $\mathrm{TN}=$ true negative, $\mathrm{FP}=$ false positive respectively.

Sensitivity and Specificity are checking accuracies of face recognition system to retrieve true positive and reject false positive images from the database.

\section{Results}

The experiment results are demonstrated using two different face image datasets. One is GT face images and another is ORL face images ${ }^{(23,24)}$. Initially, LBP and GLCM methods were tested separately, later with proposed GOL method. However, threshold has been implemented to retrieve top 15 and top 10 most similar images from GT and ORL database respectively. 


\subsection{Face image testing on Georgia Tech (GT) dataset}

A query face image is given as input to GLCM method. Figure 6 shows that system retrieves total 15 face images out of them 10 and 5 are true positive and false positive respectively.

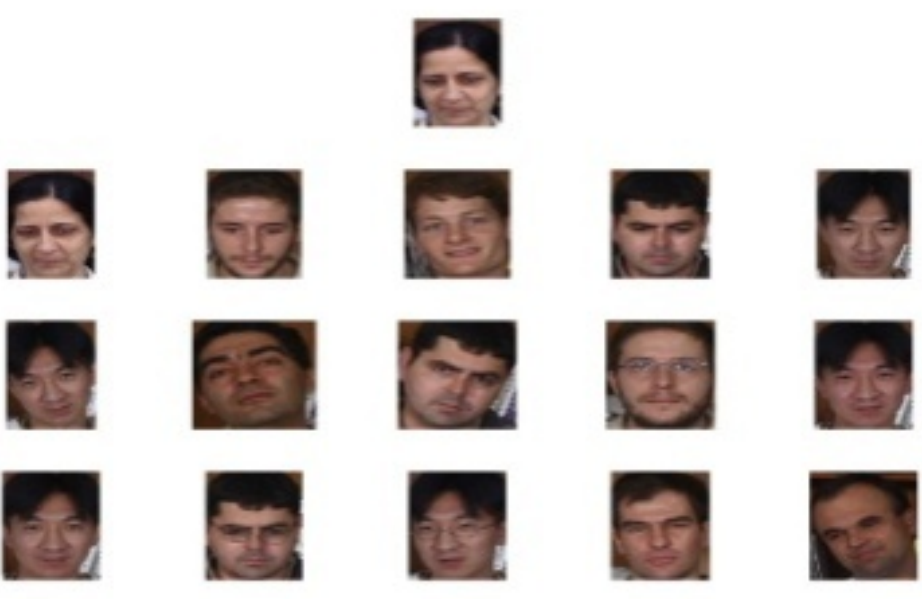

Fig 6. Image query using GLCM method
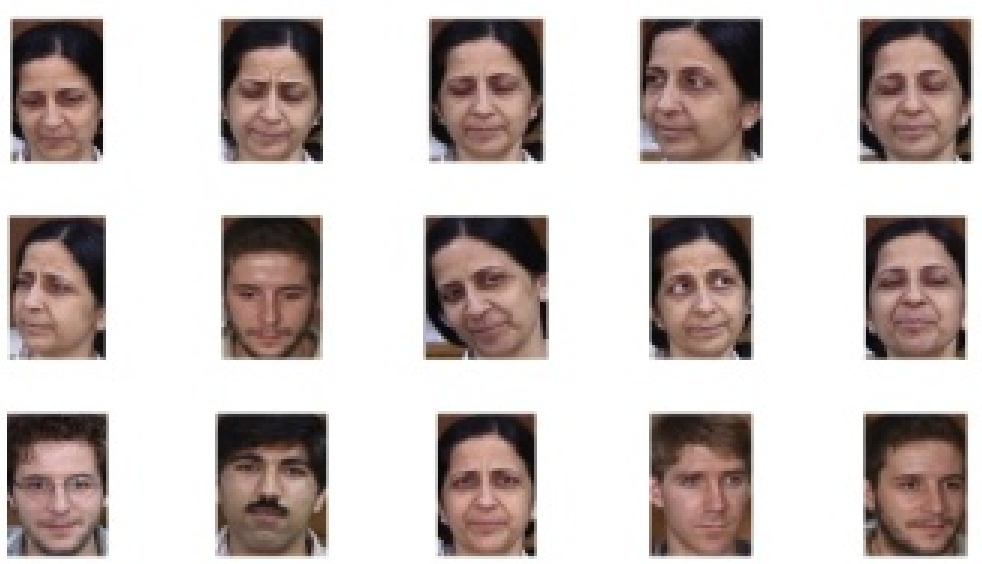

Fig 7. Image query using LBP method

The same face image is given as input using LBP method, where Figure 7 shows that it has retrieved only one relevant image out of 15 images. In Figure 8, proposed GOL method has been tested on face image which recognizes 13 accurate images out of 15 images. 


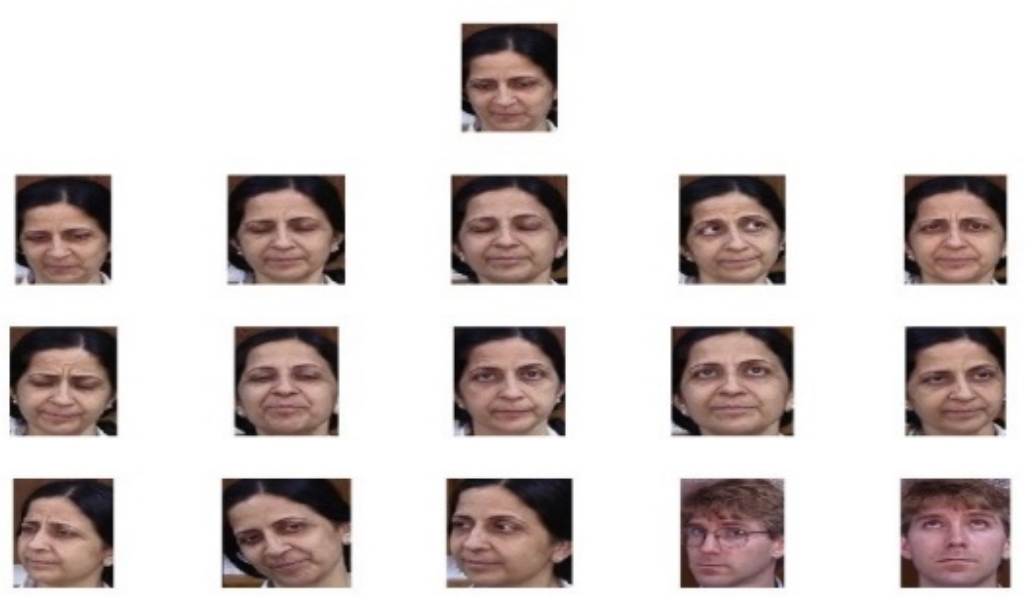

Fig 8. Image query using proposed method

\subsection{Face image testing on ORL $d$ ataset}

In this section, further investigation has been carried out using ORL dataset. Similarly, in Figure 9, input face image has been given to system using GLCM method, in which it provides 7 and 3 for true positive and false positive images out of 10 images respectively.

In figure 10 LBP method has been tested on face image. The obtained results show that it has retrieved 3 true positive and 7 false positive out of 10 images.
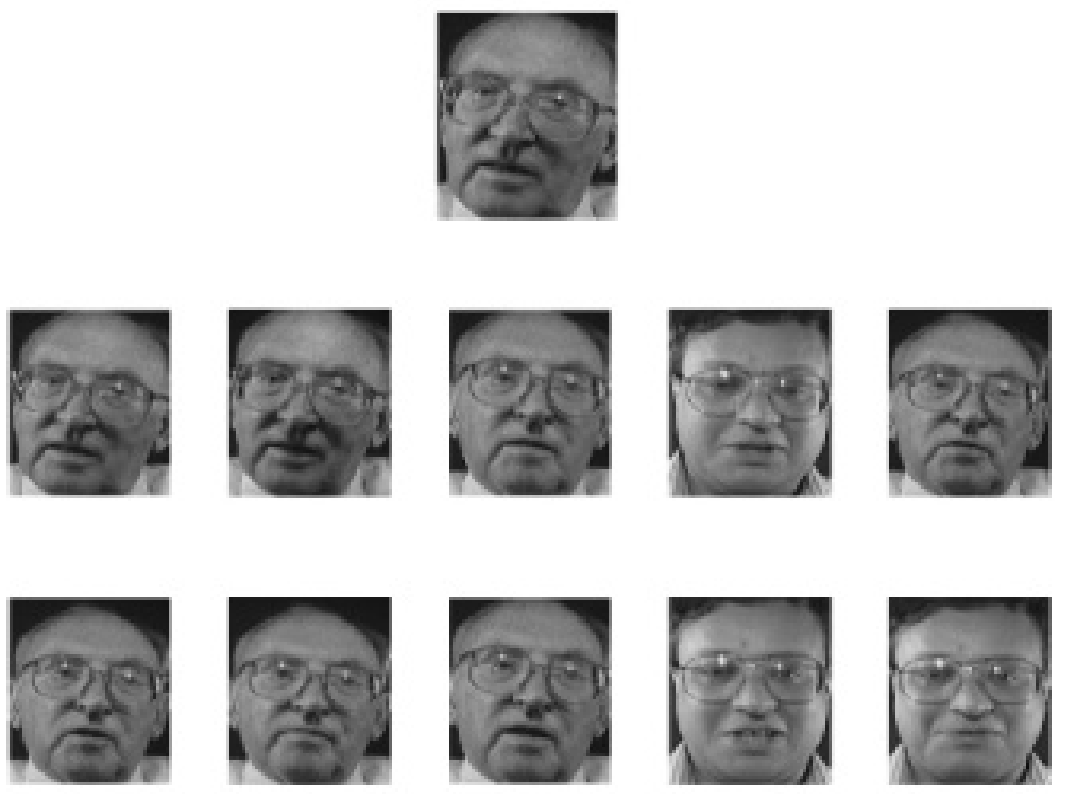

Fig 9. Image query using GLCM 

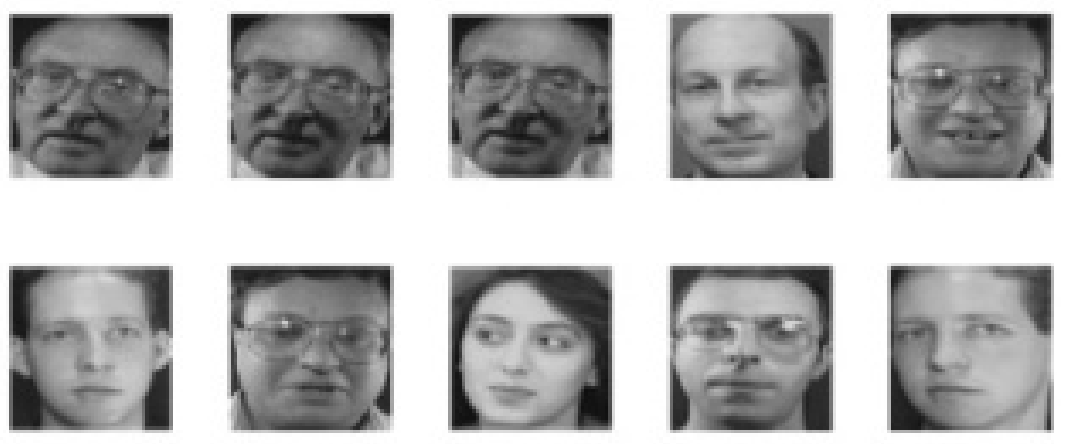

Fig 10. Image query using LBP method

Finally, the proposed GOL method has been tested, Figure 11represents results that it retrieved 10 true positive out of 10 images i.e., all are correct.
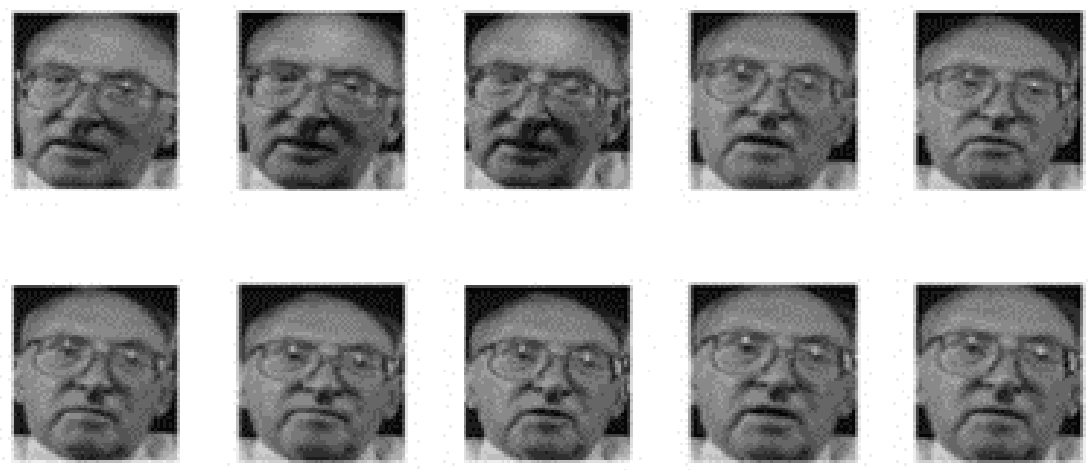

Fig 11. Proposed GOL method 


\section{Performance Evaluation}

In this section, performance of face recognition system has been evaluated using average sensitivity, average specificity and retrieval time. It can be examined from Table 1 that average sensitivity generated by GLCM and LBP on GT and ORL datasets are $41.33 \%, 30 \%$ and $46.75 \%, 41.75 \%$ respectively. The specificity of the system in terms of false positive face image rejection on GT and ORL datasets are $793.51 \%, 92.25 \%$ and $98.25 \%, 98.64 \%$ respectively. Also average time retrieval generated by each method is approximately similar.

Table 1. Performance of Face Recognition System

\begin{tabular}{llllll}
\hline \multirow{2}{*}{ Data set } & Face recognition & \multicolumn{4}{l}{ Face recognition method } \\
\cline { 2 - 5 } & accuracy & GLCM & LBP & Proposed GLCM (LBP) & Comments \\
\hline \multirow{3}{*}{ GT } & Average retrieval time & $1.306 \mathrm{sec}$ & $1.334 \mathrm{sec}$ & $1.309 \mathrm{sec}$ & Approximately equal \\
& Average sensitivity & $41.33 \%$ & $30.00 \%$ & $59.33 \%$ & Big improvement \\
& Average specificity & $93.51 \%$ & $92.25 \%$ & $95.33 \%$ & Big improvement \\
\multirow{2}{*}{ ORL } & Average retrieval time & $0.512 \mathrm{sec}$ & $0.55 \mathrm{sec}$ & $0.520 \mathrm{sec}$ & Approximately equal \\
& Average sensitivity & $46.75 \%$ & $41.75 \%$ & $59.75 \%$ & Big improvement \\
& Average specificity & $98.61 \%$ & $98.48 \%$ & $98.95 \%$ & Big improvement \\
\hline
\end{tabular}

The average sensitivity and specificity obtained through proposed GOL method are 59.33\%, 59.75\% and 95.33\%, 98.95\% respectively, which are quite higher than single GLCM and LBP method. However, average retrieval time is also equal.

\subsection{Comparative analysis on databases}

In this section, we have performed comparative analysis of face recognition over GT and ORL facial dataset. The Figures 12 and 13 represent generated graphs of average specificity, average sensitivity on GT dataset. In addition, graph is divided into 10 face classes having each 10 subject images. Visual analysis reveals that proposed GOL technique better as compared to GLCM and LBP method.

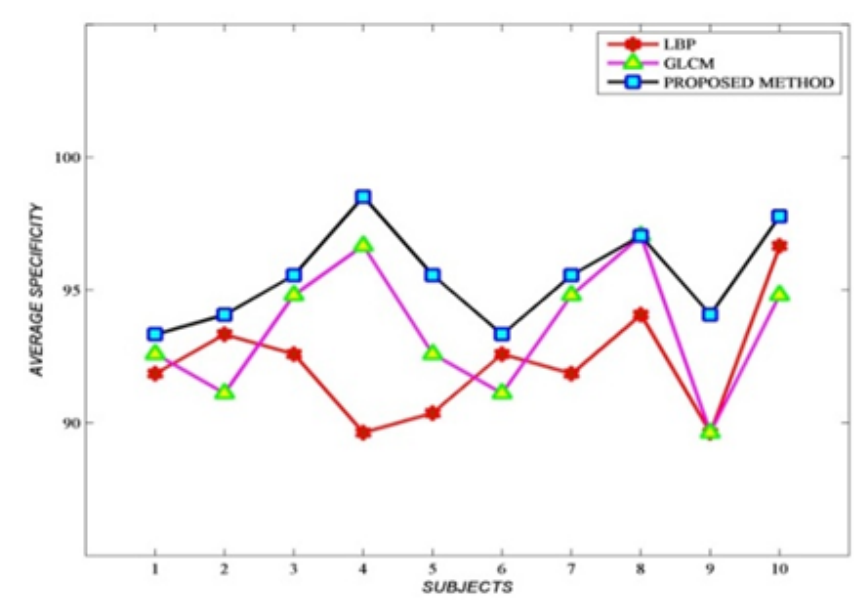

Fig 12. Average specificity of proposed, LBP and GLM method

However, Figure 14 depicts that efficiency of the proposed GOL method in terms of average retrieval time on GT dataset is approximately similar.

The Figures 15 and 16 show generated graphs on ORL facial database. Graph is divided into 40 face classes each having 20 subject images. Visual analysis provides that the accuracy of GLCM and LBP method in terms of average sensitivity and specificity is quite lower than compared to proposed GOL technique.

However, the average retrieval time of GOL method as shown in Figure 17 is comparatively similar to GLCM and LBP method. 


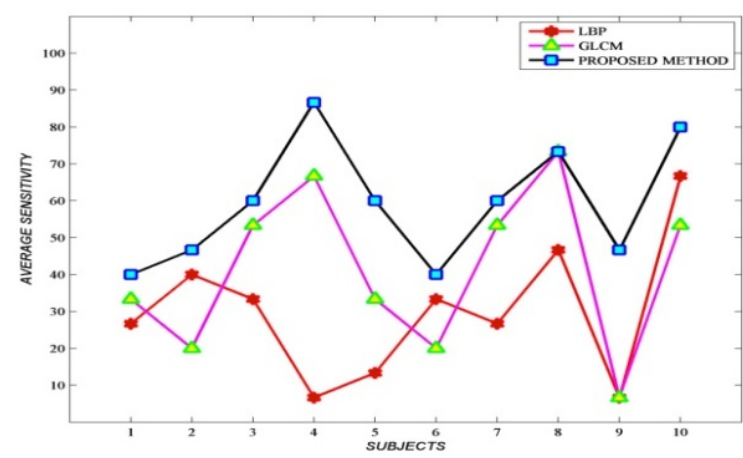

Fig 13. Average sensitivity of proposed, LBP and GLCM method on GT database

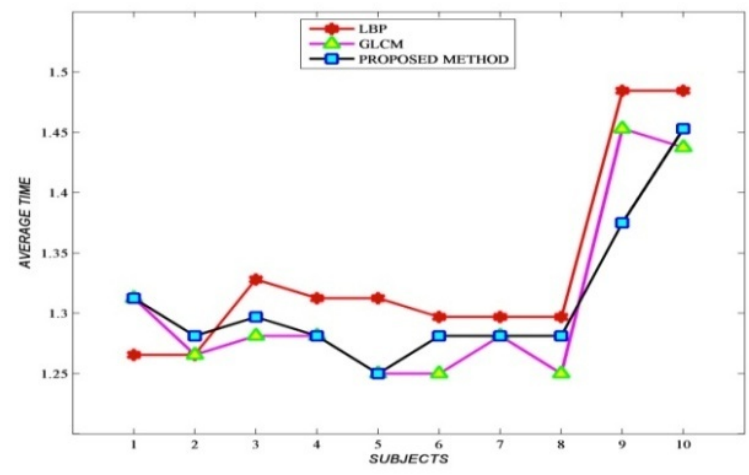

Fig 14. Average retrieval time of proposed, LBP and GLM method on GT database

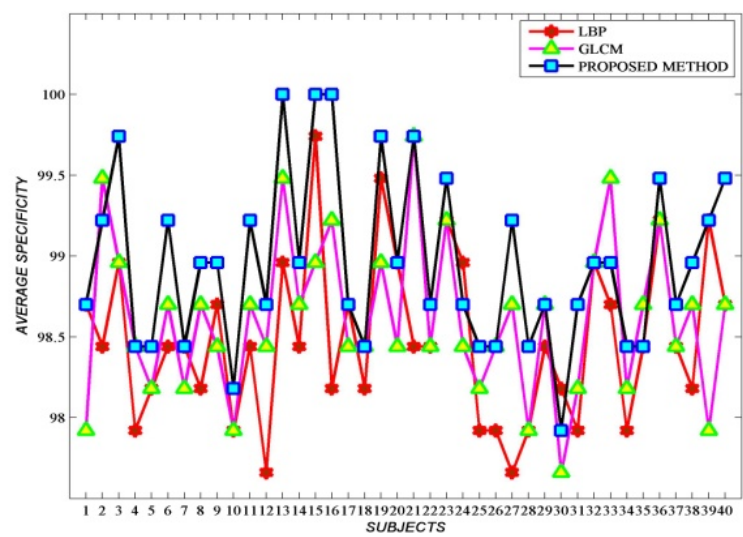

Fig 15. Average specificity of proposed, LBP and GLM method on ORL database 


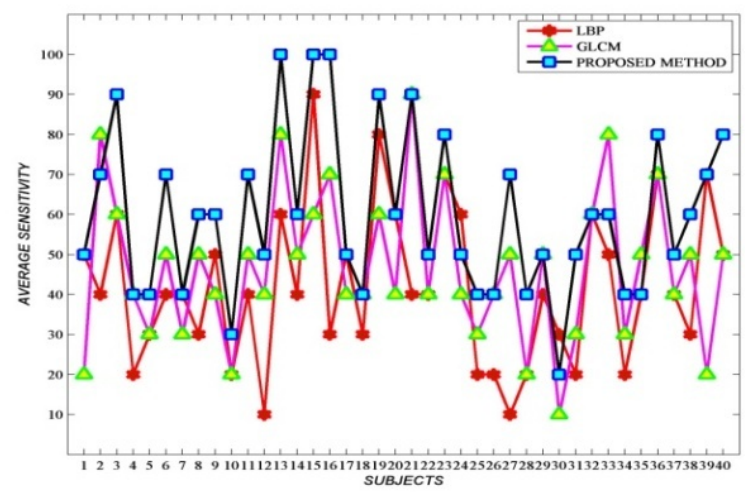

Fig 16. Average sensitivity of proposed, LBP and GLCM method on ORL database

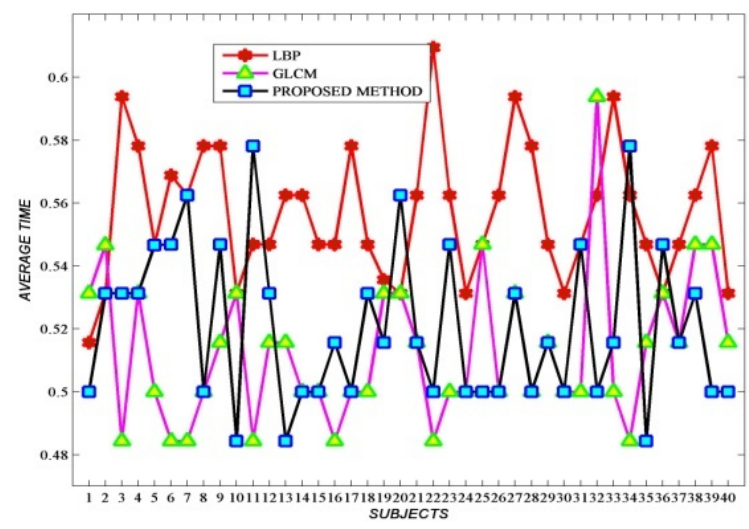

Fig 17. Average retrieval time of proposed, LBP And GLM method on GT database

\section{Conclusion and Future Work}

Feature extraction remains most challenging in the context of face recognition since last few decades. In this paper, face recognition system was developed using a new method called GOL texture features for ORL and GT facial images databases. In order to evaluate the proposed system separate investigation was performed using GLCM and LBP for similar databases. The performance of each face recognition method was judged through their average specificity, average sensitivity and average retrieval time. After comparative analysis accuracy of the proposed GOL method was quite higher than separate GLCM and LBP methods. The obtained results of GLCM and LBP were also compared with the results of the proposed method and the results reveal that the proposed method is a better method for face recognition system.

\section{References}

1) Numitha MN, Noorain T, Amulya S, Patil, Navyashree HV, Nagalakshmi TS, et al. Face Recognition and IoT Based Smart Lock Access System. International Journal of Scientific Research in Computer Science, Engineering and Information Technology (IJSRCSEIT). 2018;4(6):701-704.

2) Baqeel H, Saeed S. Face detection authentication on Smartphones: End Users Usability Assessment Experiences. In: 2019 International Conference on Computer and Information Sciences (ICCIS). 2019;p. 1-6.

3) Kaur P, Krishan K, Sharma SK, Kanchan T. Facial-recognition algorithms: A literature review. Medicine, Science and the Law. 2020;60(2):131-139. doi:10.1177/0025802419893168.

4) Hassaballah M, Aly S. Face recognition: challenges, achievements and future directions. IET Computer Vision. 2015;9(4):614-626. doi:10.1049/ietcvi.2014.0084.

5) Zhao W, Chellappa R, Phillips PJ, Rosenfeld A. Face Recognition: A Literature Survey. ACM Computing Surveys. 2003;35:399-458.

6) Lal M, Kumar K, Hussain R, Maitlo A. Hidayatullah Shaikh. Study of Face Recognition Techniques: A Survey. J] International Journal of Advanced Computer Science and Applications. 2018;9(6):42-49. 
7) Ahonen T, Hadid A, Pietikainen M. Face Description with Local Binary Patterns: Application to Face Recognition. IEEE Transactions on Pattern Analysis and Machine Intelligence. 2006;28(12):2037-2041. doi:10.1109/tpami.2006.244.

8) Li SZ, Chu R, Liao S, Zhang L. Illumination Invariant Face Recognition Using Near-Infrared Images. IEEE Transactions on Pattern Analysis and Machine Intelligence. 2007;29(4):627-639. doi:10.1109/tpami.2007.1014.

9) Hadid A, Pietikainen M. Combining Motion and Appearance for Gender Classification from Video Sequences. 19th International Conference on Pattern Recognition. 2008;p. 1-4.

10) Pham-Ngoc PT, Jo KH. Color- Based Face Detection using Combination of Modified Local Binary Pattern and Embedded Hidden Markov Models. International Joint Conference. 2006;p. 5598-5603.

11) Eleyan A, Demirel, Hasan. Co-occurrence Based Statistical Approach for Face Recognition. 24th International Symposium on Computer and Information Sciences. 2009;p. 611-515.

12) Baek J, Kim J, Kim E. Part-based Face Detection using SLBP. 14th International Conference on Control, Automation and Systems. $2014 ;$ p. $1501-1503$.

13) Liao S, Zhu X, Lei Z. Learning Multi-scale BlockLocal Binary Patterns for Face Recognition. Advances in Biometrics Springer Book. $2007 ;$ p. 828-837.

14) Kafai M, An L, Bhanu B. Reference Face Graph for Face Recognition. IEEE Transactions on Information Forensics and Security. 2014;9(12):2132-2143. doi:10.1109/tifs.2014.2359548.

15) Xie S, Hu H. Facial expression recognition with FRR-CNN. Electronics Letters. 2017;53(4):235-237.

16) Corneanu CA, Simon MO, Cohn JF, Guerrero SE. Survey on RGB, 3D, Thermal, and Multimodal Approaches for Facial Expression Recognition: History, Trends, and Affect-Related Applications. IEEE Transactions on Pattern Analysis and Machine Intelligence. 2016;38(8):1548-1568. doi:10.1109/tpami.2016.2515606.

17) Peng F, Zhang LB, Long M. FD-GAN: Face De-Morphing Generative Adversarial Network for Restoring Accomplice's Facial Image. IEEE Access. 2019;7:75122-75131. doi:10.1109/access.2019.2920713.

18) Zhang K, Huang Y, Du Y, Wang L. Facial expression recognition based on deep evolutional spatial-temporal networks. IEEE Transactions on Image Processing. 2017;26(9):4193-4193.

19) Zhang T, Zheng W, Cui Z, Zong Y, Yan J, Yan K. A Deep Neural Network-Driven Feature Learning Method for Multi-view Facial Expression Recognition. IEEE Transactions on Multimedia. 2016;18(12):2528-2536. doi:10.1109/tmm.2016.2598092.

20) Pali V, Goswami S, Bhaiya LP. An Extensive Survey on Feature Extraction Techniques for Facial Image Processing. International Conference on Computational Intelligence and Communication Networks. 2014;p. 142-148.

21) Ojala T, Pietikäinen M, Harwood D. A comparative study of texture measures with classification based on featured distributions. Pattern Recognition. 1996;29(1):51-59. doi:10.1016/0031-3203(95)00067-4.

22) Haralick RM, Shanmugam K, Dinstein I. Textural Features for Image Classification. IEEE Transactions on Systems, Man, and Cybernetics. 1973;SMC3(6):610-621. doi:10.1109/tsmc.1973.4309314.

23) .2020. Available from: http://www.anefian.com/research/face_reco.htm.

24) . 2020. Available from: http://www.cl.cam.ac.uk/research/dtg/attarchive/facedatabase.html. 